\title{
Evolutionary history of Leishmania killicki (synonymous Leishmania tropica) and taxonomic implications
}

\author{
Dhekra Chaara 1,2,3* , Christophe Ravel ${ }^{2,3}$, Anne- Laure Bañuls ${ }^{3}$, Najoua Haouas ${ }^{1}$, Patrick Lami ${ }^{2,3}$, Loïc Talignani ${ }^{2,3}$, \\ Fouad El Baidouri ${ }^{2,3,4}$, Kaouther Jaouadi ${ }^{1}$, Zoubir Harrat ${ }^{5}$, Jean-Pierre Dedet ${ }^{2,3}$, Hamouda Babba ${ }^{1}$ \\ and Francine Pratlong ${ }^{2,3}$
}

\begin{abstract}
Background: The taxonomic status of Leishmania (L.) killicki, a parasite that causes chronic cutaneous leishmaniasis, is not well defined yet. Indeed, some researchers suggested that this taxon could be included in the L. tropica complex, whereas others considered it as a distinct phylogenetic complex. To try to solve this taxonomic issue we carried out a detailed study on the evolutionary history of L. killicki relative to L. tropica.

Methods: Thirty-five L. killicki and 25 L. tropica strains isolated from humans and originating from several countries were characterized using the MultiLocus Enzyme Electrophoresis (MLEE) and the MultiLocus Sequence Typing (MLST) approaches.

Results: The results of the genetic and phylogenetic analyses strongly support the hypothesis that L. killicki belongs to the L. tropica complex. Our data suggest that L. killicki emerged from a single founder event and that it evolved independently from L. tropica. However, they do not validate the hypothesis that L. killicki is a distinct complex. Therefore, we suggest naming this taxon L. killicki (synonymous L. tropica) until further epidemiological and phylogenetic studies justify the L. killicki denomination.
\end{abstract}

Conclusions: This study provides taxonomic and phylogenetic information on L. killicki and improves our knowledge on the evolutionary history of this taxon.

Keywords: Leishmania killicki, Leishmania tropica, Evolutionary history, Phylogeny, Isoenzymatic polymorphism

\section{Background}

Leishmaniases are neglected tropical diseases caused by Leishmania parasites and transmitted to mammals through bites by infected Phlebotomine sandflies of the genus Phlebotomus [1]. In humans, these diseases can have cutaneous $(\mathrm{CL})$, muco-cutaneous $(\mathrm{MCL})$ or visceral (VL) clinical manifestations.

Since the first description of the genus Leishmania Ross, 1903, the classification methods have considerably

\footnotetext{
*Correspondence: chaara.dhekra@yahoo.fr

'Département de Biologie Clinique B, Laboratoire de Parasitologie-Mycologie Médicale et Moléculaire (code LR12ES08), Faculté de Pharmacie, Université de Monastir, Monastir, Tunisia

2Département de Parasitologie-Mycologie, Centre National de Référence des Leishmanioses, CHRU de Montpellier, Université de Montpellier, France, 39 avenue Charles FLAHAULT, 34295 Montpellier Cedex 5, France Full list of author information is available at the end of the article
}

evolved. Indeed, between 1916 and 1987, Leishmania taxonomy followed the Linnaean classification system, mainly based on extrinsic features, such as clinical manifestations, geographical distribution, epidemiological cycles and behaviour in sandfly vectors. This method has led to the subdivision of the Leishmania genus in the two sub-genera Leishmania and Viannia [2,3].

In the eighties, the biochemical classification based on the study of the parasite isoenzymatic patterns started to be developed. This approach has evolved from the classical Adansonian to the numerical cladistic classification method that uses isoenzymes as evolutionary markers [4-8]. The description of several Leishmania complexes in the Old and New World is based on these analyses. Specifically, by using numerical phenetic and phylogenetic approaches, Rioux et al. [9] identified four main 
Leishmania groups in the Old World, while Thomaz et al. and Cupolillo et al. [10,11] defined eight complexes and two Leishmania groups in the New World.

Currently, the numerical taxonomic approach based on isoenzyme analysis is considered as the gold standard for the classification of the genus Leishmania and is routinely used for classification updates and for epidemiological studies $[12,13]$. The drawbacks of this approach are the need of bulk cultures of Leishmania parasites and its relatively poor discriminatory power. It is also time-consuming. Therefore, DNA-based techniques represent valuable alternatives for the identification and the classification of these parasites.

Since the nineties, several DNA-based approaches that target nuclear and/or kinetoplastid markers have been used for phenetic and phylogenetic studies of Leishmania, including sequencing of PCR-generated fragments (PCR-sequencing) [14], nested PCR [15], random amplified polymorphic DNA (RAPD) [16,17], single strand conformation polymorphism (SSCP) analysis $[18,19]$, multilocus sequence typing (MLST) [20], multilocus microsatellite typing (MLMT) [21,22], restriction fragment length polymorphism analysis of PCRamplified fragments (PCR-RFLP) [23,24], high-resolution melting (HRM) [25] and amplified fragment length polymorphism (AFLP) [26]. These techniques have improved our epidemiological knowledge and consequently also leishmaniasis control and treatment. Due to the wealth of new taxonomic data generated by these DNA-based approaches, it is currently debated whether the genus Leishmania classification should be revised $[27,28]$.

MLST is one of the most appropriate approaches for taxonomic studies because it provides data on the genetic variations of housekeeping genes. This approach has been increasingly used for phylogenetic investigations to understand the epidemiological and transmission features of many Leishmania complexes [20,29-33]. However, because of the complexity of this genus and the lack of studies, several taxa need to be detailed further [34].

Leishmania killicki is a recently described taxon that causes CL in Tunisia [35], Libya [36] and Algeria [37]. L. killicki taxonomic status and evolutionary history relative to $L$. tropica are based on very few studies and samples. The numerical taxonomic analysis using the Multilocus Enzyme Electrophoresis (MLEE) approach first included this parasite in the L. tropica complex $[9,38]$. However, after the revision of the Leishmania genus classification, it was considered as a separate phylogenetic complex [39]. Recently, an update study by Pratlong et al. [12] confirmed the inclusion of L. killicki within the L. tropica complex. Phenetic and phylogenetic studies using MLMT [40], PCR-sequencing [41] and MLST [31] also classified L. killicki within the L. tropica complex and suggested a closer genetic link with L. tropica from Morocco. However, these data were obtained using only seven $L$. killicki strains: two strains were analyzed by Schwenkenbecher et al. [40], two by Chaouch et al. [41] and three by El Baidouri et al. [31]. Therefore, the present study wanted to analyze by MLST a large number of L. killicki and L. tropica strains in order to precisely determine the evolutionary history and the taxonomic status of L. killicki.

\section{Methods}

\section{Origin of strains}

For this study, strains of L. killicki $(\mathrm{n}=35)$, L. tropica $(\mathrm{n}=25)$, L. major $(\mathrm{n}=1)$ and L. infantum $(\mathrm{n}=1)$ from different geographic areas and with various zymodeme patterns were included (total $=62$ strains). These strains were from human cutaneous lesions, except the $L$. infantum strain that was isolated from a patient with VL. Most strains $(n=53)$ were selected from the Cryobank of the Centre National de Référence des Leishmanioses (CNRL) (Montpellier, France) and nine L. killicki strains were collected by the team of the Laboratoire de Parasitologie - Mycologie Médicale et Moléculaire (Monastir, Tunisia) during epidemiological investigations.

Forty-eight strains, among which $34 \mathrm{~L}$. killicki strains (six from Algeria, one from Libya and 27 from Tunisia) and $14 \mathrm{~L}$. tropica strains from Morocco were analyzed by MLST for the first time during this study. The eleven remaining $L$. tropica strains were from several countries (one from Egypt, one from Greece, two from Israel, two from Jordan, three from Kenya and two from Yemen) and were previously typed by MLST. Their sequences were published in Genbank under the following accession numbers: KC158621, KC158637, KC158643, KC158677, KC158682, KC158683, KC158690, KC158696, KC158711, KC158722 and KC158761 (see [31]). One L. killicki strain (LEM163) MHOM/TN/80/LEM163 had also already been analyzed by MLST (Genbank accession number KC158820 (see [31]).

The L. major (LEM62) MHOM/YE/76/LEM62 and L. infantum (LEM75) MHOM/FR/78/LEM75 strains, previously typed by MLST, were used as outgroups [31].

\section{Isoenzymatic identification}

All studied strains were identified by MLEE, according to Rioux et al. [9], using 15 enzymatic systems: malate dehydrogenase (MDH, EC 1.1.1.37), malic enzyme (ME, EC 1.1.1.40), isocitrate dehydrogenase (ICD, EC 1.1.1.42), phosphogluconate dehydrogenase (PGD, EC 1.1.1.44), glucose-6-phosphate dehydrogenase (G6PD, EC 1.1.1.49), glutamate dehydrogenase (GLUD, EC 1.4.1.3), NADH diaphorase (DIA, EC 1.6.2.2), nucleoside purine phosphorylase 1 and 2 (NP1, EC 2.4.2.1 and 
Table 1 Details about the origin, taxon and zymodeme of the 62 strains under study

\begin{tabular}{|c|c|c|c|c|}
\hline CNRL code & WHO code & Origin & Taxon & Zymodeme \\
\hline LEM95 & MHOM/TU/79/LEM95 & Tunisia & L. killicki & MON-8 \\
\hline LEM160 & MHOM/TN/80/LEM160 & Tunisia & L. killicki & MON-8 \\
\hline LEM163 & MHOM/TN/80/LEM163 & Tunisia & L. killicki & $\mathrm{MON}-8$ \\
\hline LEM174 & MHOM/TN/80/LEM174 & Tunisia & L. killicki & MON-8 \\
\hline LEM177 & MHOM/TN/80/LEM177 & Tunisia & L. killicki & MON-8 \\
\hline LEM179 & MHOM/TN/80/LEM179 & Tunisia & L. killicki & MON-8 \\
\hline LEM180 & MHOM/TN/80/LEM180 & Tunisia & L. killicki & MON-8 \\
\hline LEM181 & MHOM/TN/80/LEM181 & Tunisia & L. killicki & $N$ \\
\hline LEM182 & MHOM/TN/80/LEM182 & Tunisia & L. killicki & MON-8 \\
\hline LEM183 & MHOM/TN/80/LEM183 & Tunisia & L. killicki & MON-8 \\
\hline LEM184 & MHOM/TN/80/LEM184 & Tunisia & L. killicki & MON-8 \\
\hline LEM185 & MHOM/TN/80/LEM185 & Tunisia & L. killicki & $\mathrm{MO}$ \\
\hline LEM186 & MHOM/TN/80/LEM186 & Tunisia & L. killicki & MON-8 \\
\hline LEM193 & MHOM/TN/80/LEM193 & Tunisia & L. killicki & MON-8 \\
\hline LEM194 & MHOM/TN/80/LEM194 & Tunisia & L. killicki & $\mathrm{MON}-8$ \\
\hline LEM904 & MHOM/TN/80/LEM904 & Tunisia & L. killicki & MON-8 \\
\hline LEM1013 & MHOM/TN/80/LEM1013 & Tunisia & L. killicki & MON-8 \\
\hline LEM4390 & MHOM/TN/2002/LSL65 & Tunisia & L. killicki & MON-8 \\
\hline LEM4741 & MHOM/TN/2004/CRE139 & Tunisia & L. killicki & MON-8 \\
\hline LEM5420 & MHOM/TN/2007/LPN306 & Tunisia & L. killicki & MON-8 \\
\hline LEM6175 & MHOM/TN/2010/MET315 & Tunisia & L. killicki & MON-8 \\
\hline LEM6226 & MHOM/TN/2004/PLC3 & Tunisia & L. killicki & MON-8 \\
\hline LEM6228 & MHOM/TN/2003/LC39 & Tunisia & L. killicki & MON-8 \\
\hline LEM6229 & M & $\mathrm{T}$ & L. & MON-8 \\
\hline LEM6230 & MHOM/TN/2006/SSC37 & Tunisia & L. killicki & MON-8 \\
\hline LEM6231 & MHOM/TN/2005/LC24 bras & Tunisia & L. killicki & MON-8 \\
\hline LEM6423 & MHOM/TN/2012/NAS12 & Tunisia & L. killicki & MON-8 \\
\hline LEM6173 & MHOM/TN/2010/MET300 & Tunisia & L. killicki & MON-317 \\
\hline LEM6227 & MHOM MN/2005/PLC5 & Libya & L. killicki & MON-8 \\
\hline LEM4995 & MHOM/DZ/2005/LIPA07 & Algeria & L. killicki & MON-301 \\
\hline LEM6404 & MHOM/DZ/2005/LIPA11 & Algeria & L. killicki & MON-301 \\
\hline LEM6416 & MHOM/DZ/2011/LIPA283 & Algeria & L. killicki & MON-301 \\
\hline LEM6418 & MHOM/DZ/2005/LIPA14 & Algeria & L. killicki & MON-301 \\
\hline LEM6420 & MHOM/DZ/2011/LIPA281 & Algeria & L. killicki & MON-301 \\
\hline LEM6421 & MHOM/DZ/2011/LIPA282 & Algeria & L. killicki & MON-301 \\
\hline LEM1623 & 9/LEI & Morocco & L. tropica & MON-102 \\
\hline LEM1663 & MHOM/MA/89/LEM1663 & Morocco & L. tropica & MON-102 \\
\hline LEM2017 & MHOM/MA/90/LEM2017 & Morocco & L. tropica & MON-102 \\
\hline LEM5276 & MHOM/MA/2000/INHW02 & Morocco & L. tropica & MON-102 \\
\hline LEM5506 & MHOM/MA/2007/INHS10 & Morocco & L. tropica & MON-102 \\
\hline LEM1591 & MHOM/MA/89/LEM1591 & Morocco & L. tropica & MON-109 \\
\hline LEM1880 & MHOM/MA/90/LEM 1880 & Morocco & L. tropica & MON-109 \\
\hline LEM1922 & MHOM/MA/89/LEM 1922 & Morocco & L. tropica & MON-109 \\
\hline LEM1879 & MHOM/MA/89/LEM 1879 & Morocco & L. tropica & MON-112 \\
\hline LEM1918 & MHOM/MA/89/LEM 1918 & Morocco & L. tropica & MON-112 \\
\hline
\end{tabular}

Table 1 Details about the origin, taxon and zymodeme of the 62 strains under study (Continued)

\begin{tabular}{lllll}
\hline LEM1778 & MHOM/MA/89/LEM1778 & Morocco & L. tropica & MON-113 \\
LEM5283 & MHOM/MA/2000/INHW19 & Morocco & L. tropica & MON-113 \\
LEM5295 & MHOM/MA/2000/INHW20 & Morocco & L. tropica & MON-113 \\
LEM3015 & MHOM/MA/95/LEM3015 & Morocco & L. tropica & MON-264 \\
LEM0617 & MHOM/IL/80/SINGER & Israel & L. tropica & MON-54 \\
LEM955 & MHOM/YE/86/LEM955 & Yemen & L. tropica & MON-71 \\
LEM1015 & MHOM/YE/86/LEM1015 & Yemen & L. tropica & MON-71 \\
LEM1904 & MHOM/GR/88/LA615 & Greece & L. tropica & MON-114 \\
LEM1824 & MHOM/KE/86/EB103 & Kenya & L. tropica & MON-119 \\
LEM2313 & IGUG/KE/91/000 & Kenya & L. tropica & MON-119 \\
LEM2454 & MHOM/KE/92/EB000 & Kenya & L. tropica & MON-119 \\
LEM2001 & MHOM/EG/90/LPN65 & Egypt & L. tropica & MON-137 \\
LEM3956 & MHOM/L/96/LRC-L691 & Israel & L. tropica & MON-137 \\
LEM2869 & MHOM/JO/93/JH67 & Jordan & L. tropica & MON-200 \\
LEM3322 & MHOM/JO/96/JH-88 & Jordan & L. tropica & MON-265 \\
LEM62 & MHOM/YE/76/LEM62 & Yemen & L. major & MON-26 \\
LEM75 & MHOM/FR/78/LEM75 & France & L. infantum & MON-1 \\
\hline
\end{tabular}

NP2, EC 2.4.2*), glutamate oxaloacetate transaminase 1 and 2 (GOT1 and GOT2, EC 2.6.1.1), phosphoglucomutase (PGM, EC 5.4.2.2), fumarate hydratase (FH, EC 4.2.1.2), mannose phosphate isomerase (MPI, EC 5.3.1.8) and glucose phosphate isomerase (GPI, EC 5.3.1.9).

\section{DNA extraction}

Genomic DNA from cultured parasites was extracted using the QIAamp DNA Mini Kit (Qiagen, Germany) following the manufacturer's recommendations and eluted in $150 \mu \mathrm{l}$.

\section{Analysis by Multilocus sequence typing (MLST)}

The L. killicki $(\mathrm{n}=34)$ and L. tropica $(\mathrm{n}=14)$ strains that had not been previously assessed by MLST were typed using the MLST approach based on the analysis of seven loci coding for single-copy housekeeping genes that was developed and optimized by El Baidouri et al. [31]. Genomic DNA was amplified by real-time PCR using the SYBR Green method (Light cycler 480 II, Roche). The amplified products were sequenced on both strands (Eurofins MWG Operon, Germany) and the obtained sequences were aligned and checked in both directions using the CodonCode Aligner software, v.4.0.1 (Codon Code Co., USA). For each strain, polymorphic sites (PS) and ambiguous positions corresponding to heterozygous sites (HS) were identified in each locus using the same software. The DnaSP software v.5 [42] was used to calculate the number of haplotypes from the concatenated sequences. 
Table 2 Isoenzyme patterns for the 15 enzyme systems of the four Leishmania killicki zymodemes

\begin{tabular}{|c|c|c|c|c|c|c|c|c|c|c|c|c|c|c|c|c|}
\hline \multirow[t]{2}{*}{ Taxon } & \multirow[t]{2}{*}{ Zymodeme } & \multicolumn{15}{|c|}{ Enzyme profiles } \\
\hline & & $\mathrm{MDH}$ & ME & ICD & PGD & G6PD & GLUD & DIA & NP1 & NP2 & GOT1 & GOT2 & PGM & $\mathrm{FH}$ & MPI & GPI \\
\hline \multirow[t]{4}{*}{ L. killicki } & MON-317 & 100 & 100 & 100 & 93 & 82 & 110 & 100 & 300 & 100 & 127 & 90 & 100 & 110 & 110 & 76 \\
\hline & MON-8 & 100 & 100 & 100 & 93 & 82 & 110 & 100 & 300 & 100 & 127 & 90 & 100 & 100 & 110 & 76 \\
\hline & MON-301 & 112 & 93,66 & 100 & 93 & 82 & 110 & 100 & 300 & 100 & 140 & 85 & 100 & 100 & 110 & 76 \\
\hline & MON-306 & 112 & 100 & 100 & 93 & 82 & 110 & 100 & 300 & 100 & 140 & 85 & 100 & 100 & 110 & 76 \\
\hline
\end{tabular}

Phylogenetic relationships were inferred using a Bayesian approach implemented with the MrBayes software v. 3.2.3 [43]. The concatenated duplicated sequence alignments of the seven loci for the 32 Leishmania strains representing all the identified haplotypes and the two outgroup strains $(n=34$ in total) were used to run two independent chains for 10,000,000 generations each and trees sampled every 1000 generations. The burn-in period was set to 200,000 generations to fit the first $20 \%$ of the analyses. Analyses were conducted using the General time reversible model of substitution with a proportion of invariable sites and gamma distribution estimated by the $\operatorname{program}(\mathrm{GTR}+\mathrm{G}+\mathrm{I})$.

The chain convergence was assessed using the average standard deviation of split frequencies (ASDSF). If two runs converge onto the stationary distribution, the ASDSF is expected to approach zero, reflecting the fact that the two tree samples become increasingly similar. An average standard deviation below 0.01 is thought to be a very good indication of convergence (below 0.004 in our analysis). The consensus tree was constructed using 1000 trees sampled from the stationary phase. The MEGA 5.10 software [44] was used to identify amino acid variations between $L$. killicki and L. tropica.

\section{Results}

Isoenzymatic identification of Leishmania strains

Among the 62 strains under study, 53 had been previously characterized by MLEE at the Centre National de

\begin{tabular}{|c|c|c|c|}
\hline Locus & Length (bp) & $\begin{array}{l}\text { No of PS } \\
\text { (\% of length) }\end{array}$ & $\begin{array}{l}\text { No of HS } \\
\text { (\% of length) }\end{array}$ \\
\hline 03.0980 & 678 & $18(2,65 \%)$ & $11(1,62 \%)$ \\
\hline 04.0580 & 711 & $15(2,1 \%)$ & $9(1,26 \%)$ \\
\hline 10.0560 & 636 & $12(1,88 \%)$ & $5(0,78 \%)$ \\
\hline 12.0010 & 579 & $12(2,07 \%)$ & $4(0.69 \%)$ \\
\hline 14.0130 & 642 & $11(1,71 \%)$ & $8(1,24 \%)$ \\
\hline 31.0280 & 810 & $21(2,6 \%)$ & $16(1,97 \%)$ \\
\hline 31.2610 & 486 & $6(1,23 \%)$ & $6(1,23 \%)$ \\
\hline Concatenated & 4542 & 95 (2,09\%) & $59(1,3 \%)$ \\
\hline
\end{tabular}

PS: polymorphic sites, HS: heterozygous sites.
Référence des Leishmanioses. The nine strains collected by the team of the Laboratoire de Parasitologie Mycologie Médicale et Moléculaire (Monastir, Tunisia) were identified for the first time in this study using the same technique [12,35-38,45-47]. Nevertheless, all the strains were analyzed again by MLEE at the Centre National de Référence des Leishmanioses (Montpellier, France).

Seventeen zymodemes were identified: three for $L$. kill$i c k i, 12$ for $L$. tropica and a single zymodeme for each L. major and L. infantum strain (Table 1). For L. killicki, besides the two known zymodemes MON-8 $(n=28)$ and MON-301 $(n=6)$, a new zymodeme (MON-317) was characterized in a single Tunisian strain (LEM6173: MHOM/TN/2010/MET300). The zymodeme MON-317 differed from MON-8 by only a single enzyme (FH) [35]. On the other hand, the MDH, ME, GOT1, GOT2 and $\mathrm{FH}$ profiles were different in the zymodemes MON-317 and MON-301 [37], and the MDH, GOT1, GOT2 and $\mathrm{FH}$ profiles allowed discriminating between MON-317 and MON-306 (a zymodeme described in Algeria, but not included in our sample collection) [48] (Table 2). For $L$. tropica, all the identified zymodemes were already known [12,45]: MON-54 $(\mathrm{n}=1), \operatorname{MON}-71 \quad(\mathrm{n}=2)$, MON-102 ( $n=5), M O N-109(n=3), \operatorname{MON}-112(n=2)$, MON-113(n=3), MON-114 $(n=1), \operatorname{MON}-119(n=3)$,

Table 4 Comparison of the MLST data for the Leishmania killicki $(n=35)$ and Leishmania tropica $(n=25)$ strains at the seven loci under study

\begin{tabular}{lllll}
\hline Locus & Length (bp) & No of PS (\% of length) & $\begin{array}{l}\text { No of HS } \\
\text { (\% of length) }\end{array}$ \\
\cline { 3 - 4 } & & L. killicki* & L. tropica & L. tropica \\
\hline 03.0980 & 678 & $2(0,29 \%)$ & $16(2,36 \%)$ & $9(1,33 \%)$ \\
04.0580 & 711 & $2(0,29 \%)$ & $13(1,81 \%)$ & $7(0,98 \%)$ \\
10.0560 & 636 & $1(0,16 \%)$ & $11(1,72 \%)$ & $4(0,63 \%)$ \\
12.0010 & 579 & 0 & $12(2,07 \%)$ & $4(0,69 \%)$ \\
14.0130 & 642 & $1(0,15 \%)$ & $10(1,56 \%)$ & $7(1,09 \%)$ \\
31.0280 & 810 & $2(0,24 \%)$ & $19(2,36 \%)$ & $14(1,73 \%)$ \\
31.2610 & 486 & $3(0,61 \%)$ & $6(1,23 \%)$ & $3(0,36 \%)$ \\
Concatenated & 4542 & $11(0,24 \%)$ & $87(1,91 \%)$ & $48(1,06 \%)$ \\
\hline
\end{tabular}

S: polymorphic sites, HS: heterozygous sites. *For L. killicki PS=HS. 
Table 5 Amino acid variations in Leishmania killicki and Leishmania tropica sequences at the seven loci assessed by MLST

\begin{tabular}{|c|c|c|c|}
\hline \multirow[t]{2}{*}{ Locus } & \multirow[t]{2}{*}{ Position } & \multicolumn{2}{|c|}{ Amino acid } \\
\hline & & L. killicki & $\begin{array}{l}\text { L. tropica (number of strains/total } \\
\text { number of strains) }\end{array}$ \\
\hline \multirow[t]{4}{*}{03.0980} & 24 & G & G \\
\hline & 231 & $\mathrm{~L}$ & $L$ \\
\hline & 623 & V & $V(0.2), A(0.8)$ \\
\hline & 630 & $S$ & $S$ \\
\hline \multirow[t]{7}{*}{04.0580} & 6 & V & V \\
\hline & 36 & $\mathrm{H}$ & $H(0.96), R(0.04)$ \\
\hline & 258 & I & I (0.96), V (0.04) \\
\hline & 261 & V & $V(0.92), A(0.08)$ \\
\hline & 421 & V & $V(0.44), I(0.56)$ \\
\hline & 463 & $\mathrm{~L}$ & $\mathrm{~L}$ \\
\hline & 711 & $\mathrm{R}$ & $\mathrm{R}$ \\
\hline \multirow[t]{10}{*}{10.0560} & 24 & $\mathrm{~F}$ & $\mathrm{~F}$ \\
\hline & 42 & G & G \\
\hline & 51 & V & V \\
\hline & 54 & A & A \\
\hline & 61 & A & $S(0.6), A(0.4)$ \\
\hline & 496 & $\mathrm{D}$ & $\mathrm{D}(0.96), \mathrm{N}(0.04)$ \\
\hline & 535 & Y & $Y(0.88), N(0.12)$ \\
\hline & 545 & I & I (0.96), V (0.04) \\
\hline & 619 & $\mathrm{~T}$ & $\mathrm{~T}(0.64), \mathrm{A}(0.36)$ \\
\hline & 633 & $E$ & $E$ \\
\hline \multirow[t]{9}{*}{12.0010} & 72 & $D$ & $\mathrm{D}$ \\
\hline & 75 & G & G \\
\hline & 120 & $S$ & $S$ \\
\hline & 156 & K & K \\
\hline & 249 & $\mathrm{R}$ & $\mathrm{R}$ \\
\hline & 261 & $S$ & $S$ \\
\hline & 344 & A & $\mathrm{A}(0.56), \mathrm{V}(0.44)$ \\
\hline & 438 & $N$ & $\mathrm{~N}(0.96), \mathrm{K}(0.04)$ \\
\hline & 507 & G & G \\
\hline \multirow[t]{7}{*}{14.0130} & 77 & $\mathrm{~L}$ & L (0.88), Q (0.12) \\
\hline & 85 & $\mathrm{~S}$ & $P(0.52), S(0.48)$ \\
\hline & 246 & $E$ & $E$ \\
\hline & 303 & $\mathrm{~T}$ & $\mathrm{~T}$ \\
\hline & 364 & M & $\mathrm{V}(0.52), \mathrm{M}(0.48)$ \\
\hline & 367 & $\mathrm{R}$ & $\mathrm{R}(0.36), \mathrm{H}(0.48), \mathrm{C}(0.16)$ \\
\hline & 470 & Q & $\mathrm{R}(0.52), \mathrm{Q}(0.48)$ \\
\hline \multirow[t]{4}{*}{31.0280} & 7 & $\mathrm{~L}$ & $L(0.52), I(0.48)$ \\
\hline & 107 & N & $\mathrm{N}(0.48), \mathrm{S}(0.52)$ \\
\hline & 110 & । & I (0.48), S (0.52) \\
\hline & 120 & A & A \\
\hline
\end{tabular}

Table 5 Amino acid variations in Leishmania killicki and Leishmania tropica sequences at the seven loci assessed by MLST (Continued)

\begin{tabular}{|c|c|c|c|}
\hline & 187 & I & I (0.36),V (0.64) \\
\hline & 216 & A & A \\
\hline & 229 & $\mathrm{~T}$ & $\mathrm{~T}(0.88), \mathrm{A}(0.12)$ \\
\hline & 239 & $\mathrm{D}$ & $\mathrm{D}(0.92), \mathrm{G}(0.08)$ \\
\hline & 417 & $E$ & $E$ \\
\hline & 553 & Q & $\mathrm{Q}(0.92), \mathrm{K}(0.08)$ \\
\hline & 649 & $F$ & $F(0.92), L(0.08)$ \\
\hline & 717 & $\mathrm{~S}$ & $\mathrm{~S}$ \\
\hline & 807 & V & V \\
\hline 31.2610 & 133 & $L$ & $\mathrm{~L}$ \\
\hline & 162 & A & A \\
\hline & 210 & A & A \\
\hline & 327 & 1 & 1 \\
\hline & 368 & $L$ & $P(0.68), L(0.32)$ \\
\hline
\end{tabular}

MON-137 ( $\mathrm{n}=2)$, MON-200 $(\mathrm{n}=1)$, MON-264 $(\mathrm{n}=1)$ and MON-265 $(\mathrm{n}=1)($ Table 1$)$.

\section{Sequence analysis}

The sequences of the L. killicki $(\mathrm{n}=34)$ and L. tropica $(\mathrm{n}=14)$ strains were submitted to GenBank (accession numbers from KM085998 to KM086333). The sizes of the seven loci under study were identical to those reported by El Baidouri et al. [31], except for locus 12.0010 (only $579 \mathrm{pb}$ instead of $714 \mathrm{pb}$ ), leading to a total length of $4542 \mathrm{pb}$ for the concatenated sequences (Table 3). All chromatograms were clearly readable. Polymorphic sites (PS) and heterozygous sites (HS), which corresponded to ambiguous positions with two peaks, were easily identified. No tri-allelic site was detected.

\section{Genetic polymorphisms in L. killicki and in L. tropica}

Ninety-five (2.09\%) PS of which 59 (1.3\%) were heterozygous positions (HS) were identified in the $60 \mathrm{~L}$. killicki and $L$. tropica strains. The number of PS varied from six (1.23\%) for locus 31.2610 to 21 (2.6\%) for locus 31.0280 (Table 3).

In the L. killicki strains, 11 (0.24\%) PS were identified and they corresponded only to HS. Locus 31.2610 was the most polymorphic with three $(0.61 \%)$ PS, whereas locus 12.0010 had none. In the L. tropica strains, 87 (1.91\%) PS among which 48 (1.06\%) HS were found. The number of PS varied from six (1.23\%) for locus 31.2610 to $19(2.36 \%)$ for locus 31.0280 (Table 4).

Assessment of the presence of mutations in the seven loci under study in the L. killicki and L. tropica (heterozygous mutations were excluded from the analysis) 
identified 55 mutations of which 29 were silent substitutions and 26 resulted in altered amino acid residues (Table 5). All L. killicki mutations corresponded to a single amino acid change. Conversely, in the L. tropica strains, mutations could lead to more than one amino acid change.

\section{Phylogenetic analysis of $L$. killicki}

In total, 32 different haplotypes were identified: 10 for the $35 \mathrm{~L}$. killicki strains and 22 for the $25 \mathrm{~L}$. tropica strains. Twenty-six haplotypes were unique (eight for L. killicki and 18 for L. tropica) and the two taxa did not share any haplotype. The L. killicki MON-317 (strain LEM6173) had its own haplotype (Table 6). The Bayesian consensus tree using 32 strains representing all the identified haplotypes was constructed based on the concatenated sequences and duplicated nucleotide sites to avoid the loss of genetic information in ambiguous positions (Figure 1). The phylogenetic tree showed that $L$. killicki formed a separate group, although it belonged to the $L$. tropica complex. The L. killicki cluster showed low structuring and low polymorphism (see Figure 1). In contrast, $L$. tropica was highly polymorphic with strong structuring supported by high bootstrap values and some links with the country of origin, especially for strains from Kenya and Yemen. The larger and main clade was composed by all the Moroccan strains with the addition of other strains from other countries.

\section{Discussion}

Previous studies using a small number of strains and different molecular tools and analytic methods $[9,12,31,38,40]$ included $L$. killicki in the L. tropica complex, except the study by Rioux and Lanotte [39] in which it was considered as a separate phylogenetic complex. The present study wanted to improve the knowledge on L. killicki phylogeny and its evolutionary history relative to $L$. tropica by using a larger sample of $L$. killicki strains from different countries.

The phylogenetic analyses performed in this study confirm the position of this taxon within L. tropica in agreement with the previous biochemical and genetic findings. The close phylogenetic relationships between these taxa were also confirmed by the low number of polymorphic sites compared to those found between various Leishmania species [30,31]. The phylogenetic tree shows that $L$. killicki creates an independent group within L. tropica with high bootstrap value and no common haplotypes between them. Nevertheless, this taxon is included in the L. tropica complex and our data indicate that the species status of L. killicki is not justified. Furthermore, based on the L. tropica complex diversity and the multiple monophyletic branches in this complex, if $L$. killicki were to be considered as a species, the
Table 6 Haplotypes of Leishmania killicki and Leishmania tropica strains based on the concatenated sequences of the seven loci used for the MLST analysis

\begin{tabular}{|c|c|c|c|}
\hline Taxon & Haplotype & $\begin{array}{l}\text { Number of } \\
\text { strains }\end{array}$ & CNRL code \\
\hline \multirow[t]{12}{*}{ L. killicki } & 1 & 24 & $\begin{array}{l}\text { LEM95, LEM160, LEM163, LEM174, } \\
\text { LEM179, LEM183, LEM184, LEM186 }\end{array}$ \\
\hline & & & $\begin{array}{l}\text { LEM194, LEM904, LEM1013, } \\
\text { LEM4390, LEM4741, LEM4995, } \\
\text { LEM6226, LEM6229 }\end{array}$ \\
\hline & & & $\begin{array}{l}\text { LEM6230, LEM6231, LEM6404, } \\
\text { LEM6416, LEM6418, LEM6420, } \\
\text { LEM6421, LEM6423 }\end{array}$ \\
\hline & 2 & 3 & LEM0177, LEM0182, LEM5420 \\
\hline & 3 & 1 & LEM180 \\
\hline & 4 & 1 & LEM181 \\
\hline & 5 & 1 & LEM185 \\
\hline & 6 & 1 & LEM193 \\
\hline & 7 & 1 & LEM6173 \\
\hline & 8 & 1 & LEM6175 \\
\hline & 9 & 1 & LEM6227 \\
\hline & 10 & 1 & LEM6228 \\
\hline \multirow[t]{22}{*}{ L. tropica } & 11 & 2 & LEM1591, LEM1922 \\
\hline & 12 & 1 & LEM1623 \\
\hline & 13 & 2 & LEM1663, LEM5506 \\
\hline & 14 & 1 & LEM1778 \\
\hline & 15 & 2 & LEM1879, LEM1918 \\
\hline & 16 & 1 & LEM1880 \\
\hline & 17 & 2 & LEM2017 \\
\hline & 18 & 1 & LEM3015 \\
\hline & 19 & 1 & LEM5276 \\
\hline & 20 & 1 & LEM5295 \\
\hline & 21 & 1 & LEM5283 \\
\hline & 22 & 1 & LEM617 \\
\hline & 23 & 1 & LEM2869 \\
\hline & 24 & 1 & LEM1904 \\
\hline & 25 & 1 & LEM0955 \\
\hline & 26 & 1 & LEM1015 \\
\hline & 27 & 1 & LEM3322 \\
\hline & 28 & 1 & LEM2001 \\
\hline & 29 & 1 & LEM3956 \\
\hline & 30 & 1 & LEM1824 \\
\hline & 31 & 1 & LEM2313 \\
\hline & 32 & 1 & LEM2454 \\
\hline
\end{tabular}

L. tropica complex would be composed of many species. Therefore, we suggest calling this taxon L. killicki (synonymous $L$. tropica) as it was previously done before for $L$. chagasi (synonymous $L$. infantum) $[9,11,49,50]$. 


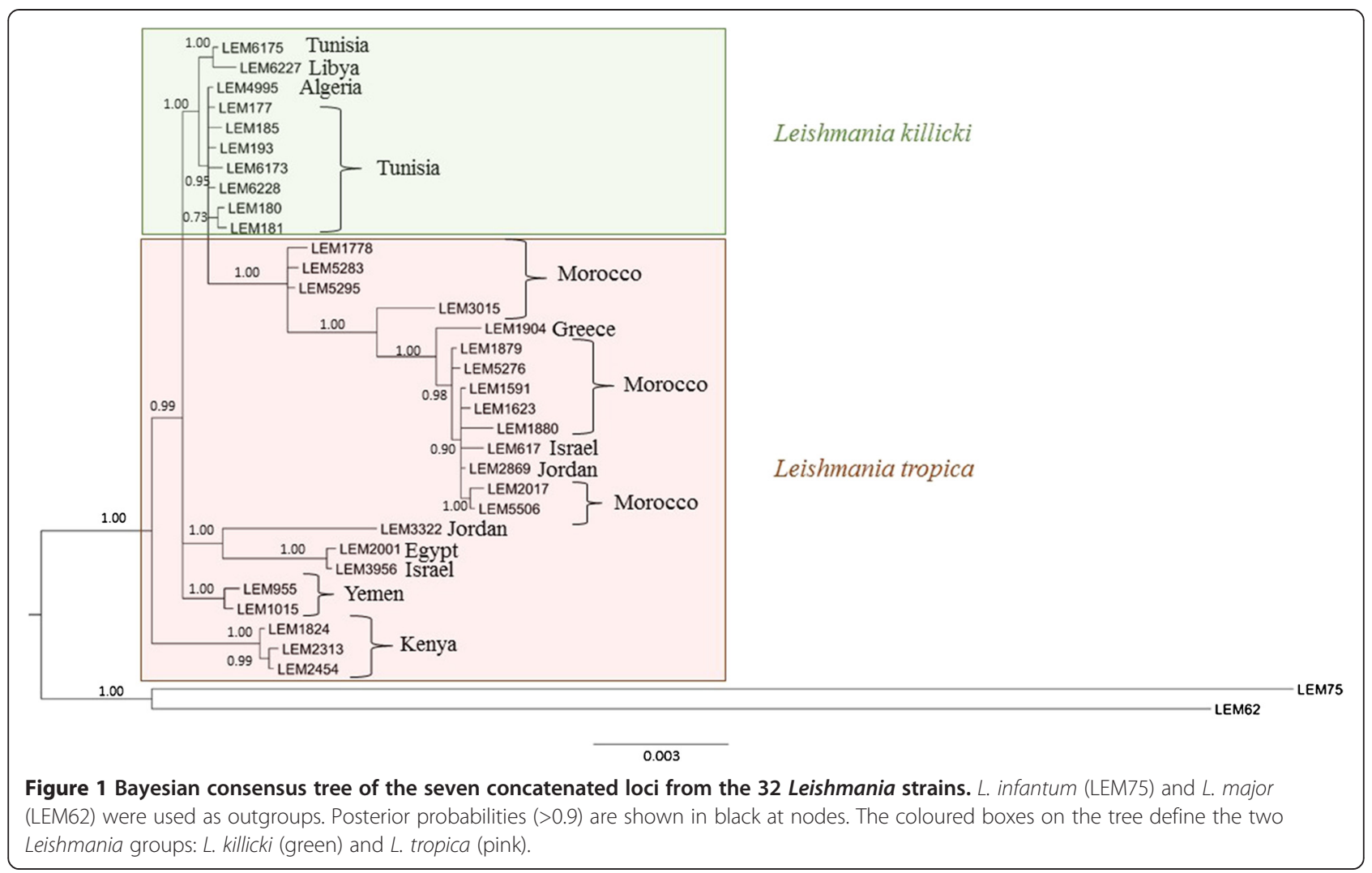

Further epidemiological and clinical studies in the different countries where this taxon has been reported will say whether the L. killicki denomination should be maintained.

From an evolutionary point of view, these data strongly suggest that L. killicki descends from L. tropica following only one founder event. This hypothesis is supported by the structure of the phylogenetic tree and by biochemical and genetic data. Indeed, the isoenzymatic characterization showed a low number of $L$. killicki zymodemes compared to those of $L$. tropica. This low polymorphism in L. killicki was confirmed by the low numbers of PS, HS and haplotypes and amino acid variations in the sequence of the different strains. The analysis of the phylogenetic tree suggests that L. killicki could have originated from an L. tropica ancestor from the Middle East. This ancestor would have separated into L. tropica in Morocco and other countries and into L. killicki in several other countries.

Finally, the lack of shared haplotypes and the identification of the new zymodeme MON-317 and its own haplotype suggest that L. killicki is now evolving independently from $L$. tropica, probably due to their different transmission cycles (zoonotic for L. killicki $[51,52]$ and both anthroponotic and zoonotic for L. tropica $[45,53,54])$.
As the L. killicki strains showed low structuring and low polymorphism, we could not determine the precise evolutionary history of this taxon and particularly the country in which it emerged for the first time. Based on the epidemiological data, the higher genetic diversity and especially the relatively high number of described cases in Tunisia compared to the other countries [35,55-57], it is likely that this taxon has emerged for the first time in Tunisia and then has spread in other North-African countries. Nevertheless, this should be further investigated.

\section{Conclusion}

The present work brings new insights into the evolutionary history of L. killicki and its taxonomic classification relative to $L$. tropica. However, more investigations need to be carried out on this model and particularly a detailed population genetics analysis to better understand the epidemiology and population dynamics of this parasite in comparison to L. tropica.

\section{Competing interests}

The authors declare that they have no competing interests.

\section{Authors' contributions}

This work was accomplished by the contribution of all authors cited: DC carried out all the technical experiments and drafted the manuscript; CR contributed to the analysis, interpretation of data and was involved in the 
revision of the manuscript; ALB participated in the analysis, interpretation of data and has contributed to the draft and revision of the manuscript; $\mathrm{NH}$ has been involved in the revision of the manuscript; PL and LT have participated in the technical experiments; FEB has contributed to data analysis; $\mathrm{KJ}$ and $\mathrm{ZH}$ have participated in sample collection; JPD was involved in the revision of the manuscript; HB directed the study; FP directed the study, revised and approved the manuscript. All authors read and approved the final manuscript.

\section{Acknowledgements}

This study was funded by the Institut Français de Coopération de Tunisie fellowship for PhD students. We thank Elisabetta Andermarcher for editing the English.

\section{Author details}

'Département de Biologie Clinique B, Laboratoire de Parasitologie-Mycologie Médicale et Moléculaire (code LR12ES08), Faculté de Pharmacie, Université de Monastir, Monastir, Tunisia. 'Département de Parasitologie-Mycologie, Centre National de Référence des Leishmanioses, CHRU de Montpellier, Université de Montpellier, France, 39 avenue Charles FLAHAULT, 34295 Montpellier Cedex 5, France. ${ }^{3}$ UMR MIVEGEC (CNRS 5290-IRD 224-Université de Montpellier), Montpellier 34394, France. ${ }^{4}$ School of Life Sciences University of Lincoln, Joseph Banks Laboratories, Green Lane, Lincoln LN6 7DL, UK. ${ }^{5}$ Laboratoire d'éco-épidémiologie Parasitaire et Génétique des Populations, Institut Pasteur d'Algérie, Dely Ibrahim, Algeria.

Received: 18 September 2014 Accepted: 21 March 2015 Published online: 01 April 2015

\section{References}

1. WHO: Control of the leishmaniases: report of a meeting of the WHO Expert Committee on the Control of Leishmaniases. World Health Organ Tech Rep; 2010: Ser 949, Geneva.

2. Safjanova VM, Sér. Protozoologie. Le problème de taxonomie chez les Leishmania. In: Les Leishmanies. Léningrad: Académie Soviétique des Sciences. Science; 1982. p. 5-109.

3. Lainson R, Shaw JJ. Evolution, classification and geographical distribution. In: Peters W, Killicki- Kendrick R, editors. The Leishmaniases Biology and medicine. London: Academie Press; 1987. p. 1-120.

4. Lanotte G, Rioux JA, Maazoun R, Pasteur N, Pratlong F, Lepart J. Application de la méthode numérique à la taxonomie du genre Leishmania Ross, 1903. A propos de 146 souches originaires de l'Ancien Monde. Utilisation des Allozymes. Corollaires épidémiologiques et phylétiques. Annals Parasitol Hum Comp. 1981:56:575-92.

5. Lanotte G, Rioux JA, Lepart J, Maazoun R, Pasteur N, Pratlong F. Contribution de la cladistique numérique à la phylétique de genre Leishmania Ross, 1903 (Kinetoplastida- Trypanosomatidae). Utilisation des caractères enzymatiques. C R Acad Sci, Paris. 1984;299:769-72.

6. Lanotte G, Rioux JA, Serres E. Approche cladistique du genre Leishmania Ross, 1903. A propos de 192 souches originaires de l'Ancien Monde. Analyse numérique de 50 zymodèmes identifiés par 15 enzymes et 96 isoenzymes. In: Rioux JA, editor. Leishmania, Taxonomie et Phylogénèse. Montpellier: IMEE/CNRS/INSERM, Montpellier; 1986. p. 269-88.

7. Le Blancq SM, Cibulkis RE, Peters W. Leishmania in the Old World. 5. Numerical analysis of isoenzyme data. Trans R Soc Trop Med Hyg. 1986;80:517-24.

8. Moreno G, Pratlong F, Velez ID, Restrepo M, Rioux JA. Individualisation du complexe Leishmania guyanensis. A propos de l'analyse enzymatique de sept zymodèmes. In: Rioux JA, editor. Leishmania, Taxonomie et phylogénèse. Montpellier: IMEEE/CNRS/INSERM; 1986. p. 165-72.

9. Rioux JA, Lanotte G, Pratlong F, Bastien P, Perieres J. Taxonomy of Leishmania. Use of isoenzymes. Suggestions for a new classification. Ann Parasitol Hum Comp. 1990;65:111-5.

10. Thomaz- Soccol V, Lanotte G, Rioux JA, Pratlong F, Martini- Dumas A Serres E. Phylogenetic taxonomy of New Word Leishmania. Annals Parasitol Hum Comp. 1993:68:104-6.

11. Cupolillo E, Grimaldi GJ, Momen H. A general classification of New World Leishmania using numerical zymotaxonomy. Am J Trop Med Hyg. 1994;50:296-311.

12. Pratlong F, Dereure J, Ravel C, Lami P, Balard Y, Serres G, et al. Geographical distribution and epidemiological features of Old World cutaneous leishmaniasis foci, based on the isoenzyme analysis of 1048 strains. Trop Med Int Health. 2009;14:1071-85.

13. Pratlong F, Lami P, Ravel C, Balard Y, Dereure J, Serres G, et al. Geographical distribution and epidemiological features of Old World Leishmania infantum and Leishmania donovani foci, based on the isoenzyme analysis of 2277 strains. Parasitology. 2013;140:423-34.

14. Van Eys GJ, Schoone GJ, Kroon NC, Ebeling SB. Sequence analysis of small subunit ribosomal RNA genes and its use for detection and identification of Leishmania parasites. Mol Biochem Parasitol. 1992;51:133-42.

15. Noyes HA, Reyburn H, Bailey JW, Smith D. A nested-PCR based schizodeme method for identifying Leishmania kinetoplast minicercle classes directly from clinical samples and its application to the study of the epidemiology of Leishmania tropica in Pakistan. J Clin Microbiol. 1998;36:2877-81.

16. Bañuls $A L$, Jonquieres $R$, Guerrini F, Le Pont F, Barrera C, Espinel I, et al. Genetic analysis of Leishmania parasites in Ecuador: are Leishmania (Viannia) panamensis and Leishmania (V.) guyanensis distinct taxa? Am J Trop Med Hyg. 1999;61:838-45.

17. Hide M, Bañuls AL, Tibayrenc M. Genetic heterogeneity and phylogenetic status of Leishmania (Leishmania) infantum zymodeme MON-1: epidemiological implications. Parasitology. 2001;123:425-32.

18. Schönian G, Schnur L, El Fari M, Oskam L, Kolesnikov AA, SokolowskaKöhler W, et al. Genetic heterogeneity in the species Leishmania tropica revealed by different PCR- based methods. Trans R Soc Trop Med Hyg. 2001;95:217-24.

19. Spanakos G, Piperaki ET, Menounos PG, Tegos N, Flemetakis A, Vakalis NC. Detection and species identification of Old World Leishmania in clinical samples using a PCR-based method. Trans R Soc Trop Med Hyg. 2008;102:46-53.

20. Mauricio IL, Yeo M, Baghaei M, Doto D, Pratlong F, Zemanova E, et al. Towards multilocus sequence typing of the Leishmania donovani complex: resolving genotypes and haplotypes for five polymorphic metabolic enzymes (ASAT, GPI, NH1, NH2, PGD). Int J Parasitol. 2006;36:575-69.

21. Oddone R, Schweynoch C, Schönian G, de Sousa CS, Cupolillo E, Espinosa D, et al. Development of a multilocus microsatellite typing approach for discriminating strains of Leishmania (Viannia) species. J Clin Microbiol. 2009:47:2818-25

22. Gouzelou E, Haralambous C, Antoniou M, Christodoulou V, Martinković F, Živičnjak T, et al. Genetic diversity and structure in Leishmania infantum populations from southeastern Europe revealed by microsatellite analysis. Parasit Vect. 2013;6:342.

23. Montalvo AM, Fraga J, Monzote L, Montano I, De Doncker S, Dujardin JC, et al. Heat-shick protein 70 PCR-RFLP: a universal simple tool for Leishmania species discrimination in the New and Old World. Parasitology. 2010;137:1159-68

24. Haouas N, Garrab S, Gorcii M, Khorchani H, Chargui N, Ravel C, et al. Development of a polymerase chain reaction-restriction fragment length polymorphism assay for Leishmania major/Leishmania killicki/Leishmania infantum discrimination from clinical samples, application in a Tunisian focus. Diagn Microbiol Infect Dis. 2010;68:152-8.

25. Talmi-Frank D, Abedelmajeed N, Schnur LF, Schönian G, Toz SO, Jaffe CL, et al. Detection and Identification of Old World Leishmania by High Resolution Melt Analysis. PLoS Negl Trop Dis. 2010;4:e581.

26. Odiwuor S, Vuylsteke M, De Doncker S, Maes I, Mbuchi M, Dujardin JC, et al. Leishmania AFLP: paving the way towards improved molecular assays and markers of diversity. Infect Genet Evol. 2011;11:960-7.

27. Schönian G, Mauricio I, Cupolillo E. Is it time to revise the nomenclature of Leishmania? Trends Parasitol. 2010;26:466-9.

28. Van der Auwera G, Fraga J, Montalvo AM, Dujardin JC. Leishmania taxonomy up for promotion. Trends Parasitol. 2011;27:49-50.

29. Zemanova E, Jirku M, Mauricio IL, Horak A, Miles MA, Lukes J. The Leishmania donovani complex: genotypes of five metabolic enzymes (ICD, $\mathrm{ME}, \mathrm{MPI}, \mathrm{G} 6 \mathrm{PDH}$, and FH), new targets for multilocus sequence typing. Int J Parasitol. 2007;37:149-60.

30. Boité MC, Mauricio IL, Miles MA, Cupolillo E. New insights on taxonomy, phylogeny and population genetics of Leishmania (Viannia) parasites based on multilocus sequence analysis. Plos Negl Trop Dis. 2012;6:e1888. doi:10.1371/journal.pntd.0001888.

31. El Baidouri F, Diancourt L, Berry V, Chevenet F, Pratlong F, Marty P, et al. Genetic structure and evolution of the Leishmania genus in Africa and Eurasia: What does MLST tell us? Plos Negl Trop Dis. 2013;7:e2255. doi:10.1371/journal.pntd.0002255. 
32. Zhang CY, Lu XJ, Du XQ, Jian J, Shu L, Ma Y. Phylogenetic and evolutionary analysis of Chinese Leishmania isolates based on multilocus sequence typing. Plos one. 2013;8:e63124. doi:10.1371/journal.pone.0063124.

33. Marlow MA, Boité MC, Ferreira GEM, Steindel M, Cupolillo E. Multilocus sequence analysis for Leishmania braziliensis outbreak investigation. Plos Negl Trop Dis. 2014;8(2):e2695. doi:10.1371/journal.pntd.0002695.

34. Bañuls AL, Hide M, Prugnolle F. Leishmania and the leishmaniases: A parasite genetic updates and advances in taxonomy, epidemiology and pathogeniecity in Humans. Adv Parasitol. 2007;64:1-109.

35. Rioux JA, Lanotte G, Pratlong F. Leishmania killicki n.sp (Kinetoplastida: Trypanosomatidae) Leishmania. Taxonomie et phylogenèse. In: Application éco-épidémiologique (Coll. Int. CNRS/INSERM, 1984). Montpellier: IMEEE; 1986. p. 139-42.

36. Aoun K, Bousslimi N, Haouas N, Babba H, El-Buni A, Bouratbine A. First report of Leishmania (L.) killicki Rioux, Lanotte and Pratlong, 1986 in Libya. Parasite. 2006;13:87-8.

37. Harrat Z, Boubidi SC, Pratlong F, Benikhlef R, Selt B, Dedet JP, et al. Description of a dermotropic Leishmania close to L. killicki (Rioux, Lanotte \& Pratlong 1986) in Algeria. Trans R Soc Trop Med Hyg. 2009;103:716-20.

38. Pratlong F, Lanotte G, Ashford RW, Rioux JA. Le complexe Leishmania tropica. A propos de l'analyse numérique de 29 souches identifiées par la méthode enzymatique. Leishmania. Taxonomie et phylogenèse. In: Applications éco-épidémiologiques (Coll. Int. CNRS/INSERM, 1984). Montpellier: IMEEE; 1986. p. 129-37.

39. Rioux JA, Lanotte G. Apport de la cladistique à l'analyse du genre Leishmania Ross, 1903 (Kinetoplastida: Trypanosomatidae). Corollaires épidémiologiques. Biosystema. 1993;8:79-80.

40. Schwenkenbecher JM, Wirth T, Schnur LF, Jaffe CL, Schallig H, Al-Jawabreh A, et al. Microsatellite analysis reveals genetic structure of Leishmania tropica. Int J Parasitol. 2006;36:237-46.

41. Chaouch M, Fathallah-Mili A, Driss M, Lahmadi R, Ayari C, Guizani I, et al. Identification of Tunisian Leishmania spp. by PCR amplification of cysteine proteinase B (cpb) genes and phylogenetic analysis. Acta Trop. 2013;125:357-65.

42. Librado P, Rozas J. DnaSP v5: a software for comprehensive analysis of DNA polymorphism data. Bioinformatics. 2009;25:1451-2.

43. Ronquist F, Teslenko M, van der Mark P, Ayres DL, Darling A, Höhna S, et al. MrBayes 3.2: efficient Bayesian phylogenetic inference and model choice across a large model space. Syst Biol. 2012;61:539-42.

44. Tamura K, Peterson D, Peterson N, Stecher G, Nei M, Kumar S. MEGA5: molecular evolutionary genetics analysis using maximum likelihood, evolutionary distance, and maximum parsimony methods. Mol Biol Evol. 2011;28:2731-9.

45. Pratlong F, Rioux JA, Dereure J, Mahjour J, Gallego M, Guilvard E, et al. Leishmania tropica in Morocco. IV-Intrafocal enzyme diversity. Ann Parasitol Hum Comp. 1991;66:100-4.

46. Haouas N, Chargui N, Chaker E, Ben Said M, Babba H, Belhadj S, et al. Anthroponotic cutaneous leishmanisis in Tunisia: Presence of Leishmania killicki outside its original focus of Tataouine. Trans R Soc Trop Med Hyg. 2005;99:499-501.

47. Haouas N, Chaker E, Chargui N, Gorcii M, Belhadj S, Kallel K, et al. Geographical distribution updating of Tunisian leishmaniasis foci: About the isoenzymatic analysis of 694 strains. Acta Trop. 2012;124:221-8.

48. Mansouri R, Pratlong F, Bachi F, Hamrioui B, Dedet JP. The first isoenzymatic characterizations of the Leishmania strains responsible for cutaneous leishmaniasis in the Area of Annaba (Eastern Algeria). Open Conf proceed J. 2012;3(Suppl 2-M2):6-11.

49. Momen H, Gabriel Grimaldi JR, Leonidas MD. Leishmania infantum, the aetiological agent of American visceral leismaniasis (AVL)? Mem Inst Oswaldo Cruz. 1987:82:447-8

50. Mauricio IL, Stothard JR, Miles MA. The strange case of Leishmania chagasi. Parasitol Today. 2000;16:188-9.

51. Jaouadi K, Haouas N, Chaara D, Gorcii M, Chargui N, Augot D, et al. First detection of Leishmania killicki (Kinetoplastida, Trypanosomatidae) in Ctenodactylus gundi (Rodentia, Ctenodactylidae), a possible reservoir of human cutaneous leishmaniasis in Tunisia. Parasite Vector. 2011;11:159.

52. Bousslimi N, Ben-Ayed S, Ben-Abda I, Aoun K, Bouratbine A. Natural infection of North African gundi (Ctenodactylus gundi) by Leishmania tropica in the focus of cutaneous leishmaniasis, Southeast Tunisia. Am J Trop Med Hyg. 2012;86:962-5.
53. Sang DK, Njeru WK, Ashford RW. A zoonotic focus of cutaneous leishmaniasis due to Leishmania tropica at Utut, Rift Valley Province Kenya. Trans R Soc Trop Med Hyg. 1994;88:35-7.

54. Svobodova M, Votypka J, Peckova J, Dvorak V, Nasereddin A, Baneth G, et al. Distinct transmission cycles of Leismania tropica in 2 adacent foci, Northern Israel. Emerg Infect Dis. 2006;12:1860-8.

55. Chaffai M, Ben Rachid MS, Ben Ismail R. Formes clinico-épidémiologiques des Leishmanioses cutanées en Tunisie. Ann Dermatol Venereol. 1988;115:1255-60.

56. Aoun K, Ben Abda I, Bousslimi N, Bettaieb J, Siala E, Ben Abdallah R, et al Caractérisation comparative des trois formes de leishmaniose cutanée endémique en Tunisie. Ann Dermatol Venereol. 2012;139:452-8.

57. Jaouadi K, Depaquit J, Haouas N, Chaara D, Gorcii M, Chargui N, et al. Twenty-four new human cases of cutaneous leishmaniasis due to Leishmania killicki in Metlaoui, southwestern Tunisia: probable role of Phlebotomus sergenti in the transmission. Acta Trop. 2012;122:276-83.

\section{Submit your next manuscript to BioMed Central and take full advantage of:}

- Convenient online submission

- Thorough peer review

- No space constraints or color figure charges

- Immediate publication on acceptance

- Inclusion in PubMed, CAS, Scopus and Google Scholar

- Research which is freely available for redistribution 